\title{
Interrogation unit for fiber Bragg grating sensors that uses a slanted fiber grating
}

\author{
César Jáuregui, Antonio Quintela, and José Miguel López-Higuera \\ Grupo de Ingeniería Fotónica, Departamento Transports Elèctrics Interurbans S.A., University of Cantabria, \\ Avenida Los Castros s/n, c.p. 39005 Santander, Spain
}

Received April 15, 2003

\begin{abstract}
A novel interrogation technique for fiber Bragg gratings is presented. It is based on measuring the characteristics of the outgoing near-field radiation from a tilted fiber grating. This scheme was experimentally checked and was found to offer a wide wavelength interrogation range as well as a good linearity, stability, and wavelength resolution. (c) 2004 Optical Society of America

OCIS codes: $120.0120,050.0050,250.0250,280.0280,060.0060$.
\end{abstract}

Fiber Bragg grating (FBG) technology is reaching maturity but, although it offers good characteristics ${ }^{1}$ (e.g., a dielectric nature, small size and low weight, multiplexing capabilities) that make it suitable for civil structure monitoring applications, its use is not yet widespread. Among the possible reasons for this are the reluctance of civil engineers and other potential users regarding new technologies and the relatively high price of these transducers compared with traditional ones. However, none of these reasons completely justifies the scarce market penetration of this technology. Thus, more causes should be looked for to explain this situation. One cause, possibly the most important, has to do with the interrogation unit. Up to now, proposed FBG schemes and commercially available units have exhibited important problems that limit their practical use: fragility and environmental dependence exhibited by schemes such as interferometric $\mathrm{FBGs},{ }^{2}$ a low wavelength interrogation range, lack of wavelength multiplexing capabilities that characterize lateral filtering techniques, ${ }^{3}$ and the low measurement speed of sweeping techniques (based on either lasers or tuning filters). ${ }^{4}$ In this Letter a novel interrogation scheme based on measurement with a photodetector linear array of near-field radiation outcoupled by a tilted FBG is presented. This technique has the potential to overcome most of the problems of the already proposed techniques: It can lead to a robust and compact interrogation unit suitable for in-field use; it has a wide wavelength interrogation range that, to our knowledge, doubles the widest reported up to now; it is able to simultaneously extract information out of many multiplexed gratings; and it has the potential of reaching interrogation speeds that would allow dynamic measurements.

As mentioned above, the principle of operation of the proposed technique is based on a photodetector linear array that measures the near-field radiation outcoupled by an in-fiber tilted grating when it is illuminated by light reflected by sensing FBGs. By performing an analysis of the characteristics of this radiation, one can infer the wavelengths of the light from which it originated. This operating principle is shown schematically in Fig. 1 . It can be seen that the heart of the system is a tilted and chirped FBG placed parallel to a linear array at a distance $d$ of a few millimeters. This leads to a quite compact and potentially small-sized interrogation unit.

In Fig. 2 the near-field radiation image (measured at a distance of $4.775 \mathrm{~mm}$ from the tilted grating) obtained with a 1.28-cm-long, 512-element photodetector array (Hamamatsu G8161-512S) when a 1-cm-long, $34^{\circ}$ tilt, chirped tilted grating is illuminated by 1280-nm light can be seen. This tilted grating had a linearly chirped period that ranged from 0.525 to $0.559 \mu \mathrm{m}$. This configuration of parameters is the same that will be used below for interrogation of FBGs. As highlighted schematically in Fig. 2, there are two different effects that contribute to the conformation of the radiation shape: coupling to radiation modes (as defined in coupling-mode theory) and cladding resonances. The first gives rise to a smooth, quasi-Gaussian tendency on top of which sharp peaks (formed as a result of the latter effect) can be found. These peaks occur when part of the light gets trapped in the cladding on its way to the outer medium. As can be appreciated from Fig. 2 the radiation is very wide, which means that whenever there are multiple sensing FBGs their near fields will overlap, and so a composite image will be detected at the array. Thus, a specific algorithm should be developed to decompose this new complex image into its individual near-field radiation corresponding to each of the sensing FBGs. This algorithm uses adaptive

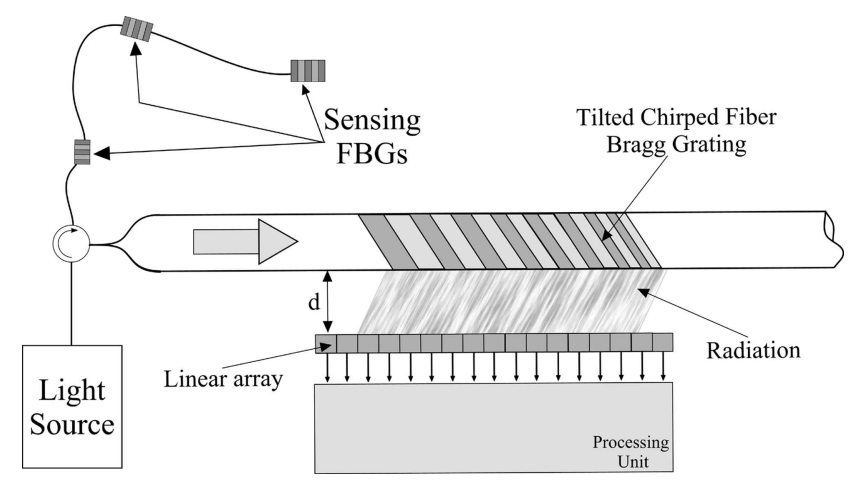

Fig. 1. Schematic diagram of the proposed interrogation technique. 


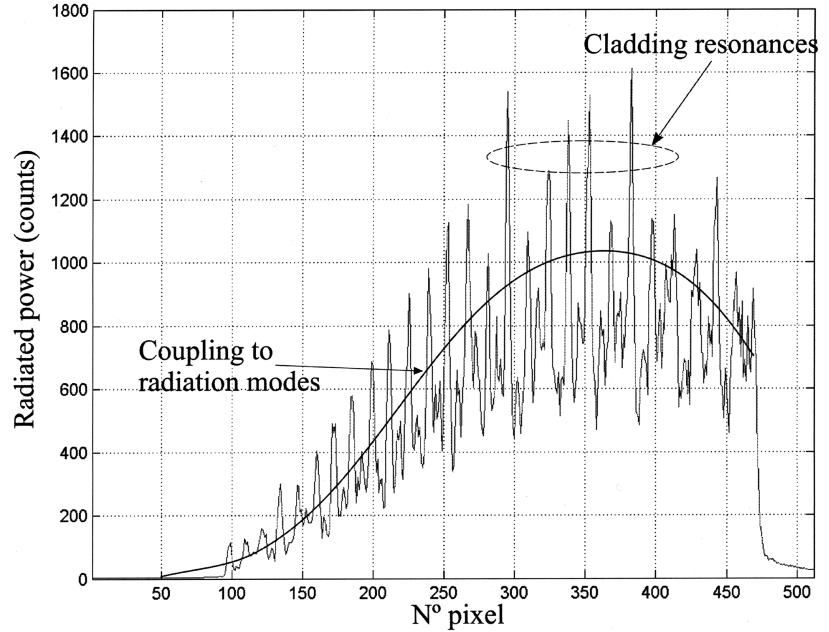

Fig. 2. Image of the near-field radiation obtained by the photodetector linear array.

filtering techniques, specifically a modified version of the Kalman filter. The operating principle of such a demultiplexing technique is based on the fact that the radiation shape emitted by the tilted FBG (for instance, that shown in Fig. 2) is unique for each wavelength, and so it constitutes a fingerprint for that parameter. Therefore, when one has a database of these fingerprints and uses appropriate signal processing, the individual shapes can be recovered from the composite image. Then, by measuring the characteristics of these individual shapes, one can infer the wavelength of each FBG.

One of the key features to be measured in each near-field radiation is its degree of spatial shift (which we understand to be the displacement of the peak radiation). This is due to the fact that whenever the Bragg wavelength is changed the radiation, as can be seen from Fig. 2, suffers a shift toward the left (if the wavelength is increased) or the right (if it is decreased). Also, because of the chirp this spatial shift is greatly magnified, as shown in Fig. 3, which justifies the choice of a chirped tilted FBG. In Fig. 3 there is a comparison between the two effects that contribute to the displacement: the wavelength dependence of the radiation output angle $\mathrm{e}^{5}$ and the chirp rate. The first of these effects is widely known and, thus, requires no further discussion, but the second should be clarified. In a tilted and chirped grating, as can be seen from Fig. 2, the radiation shape follows a quasi-Gaussian shape whose maximum is at a point that is highly wavelength dependent. This dependence arises from the fact that each subgrating (which is the way we refer to different parts of the grating in which the period can be considered constant) within the chirped grating outcouples light with maximum efficiency at a certain wavelength. Thus, having a structure that distributes these subgratings along a line (as the tilted chirped grating does) means that whenever the incident wavelength changes the peak of maximum radiated power will shift. Because of this property an increment of nearly five times in the spatial shift (for the chirp rate of the tilted FBG used) with respect to that corresponding to the output angle's effect can be obtained. This increment is shown in Fig. 3, where the displacement due to the chirp effect and the displacement due to the wavelength dependence of the output angle are compared.

As can be deduced from the explanation of the operating principle above, the technique will work properly as long as there is radiation from the tilted FBG. Thus, wavelength interrogation ranges of $160 \mathrm{~nm}$ and even greater ranges can easily be obtained. ${ }^{6}$

To evaluate the performance of the proposed scheme experimentally we implemented the system illustrated in Fig. 1. A superluminescent diode (SLD-1300, Fiberon) was used as the broadband light source. Three different 0.3-nm-wide FBGs centered at 1301.7, 1308.5 , and $1313.7 \mathrm{~nm}$ were employed as transducers, the second being attached to a pulling setup that allowed for calibrated elongations.

With the system described above, several experiments were carried out for evaluating different aspects related to the performance of the technique. The first, the results for which are shown in Fig. 4, involved applying an increasing strain to the FBG attached to the pulling setup while keeping the rest unperturbed. In Fig. 4 the responses of the three sensing FBGs obtained with the proposed scheme (when the integration time of the linear array was set to $6 \mathrm{~ms}$ and the analog-digital converter was 12 bits) are shown. As can be seen from Fig. 4, the linearity of the response is high (the subtle nonlinearity is a direct consequence of the interrogation algorithm). Also, the accuracy in determining the wavelength shift was good. In an experiment such as the one reported here, it would be expected that the responses of the unperturbed FBGs would remain constant and at a null wavelength shift. Nevertheless, a fictional wavelength shift can be observed in both responses (rather large in this example for illustrative purposes). This shift is due to one of the side effects of the algorithm developed to accomplish the decomposition of the images: the cross talk. Preliminary investigations show that this effect can be greatly reduced (below the $1 \%$ for the gratings used in these experiments)

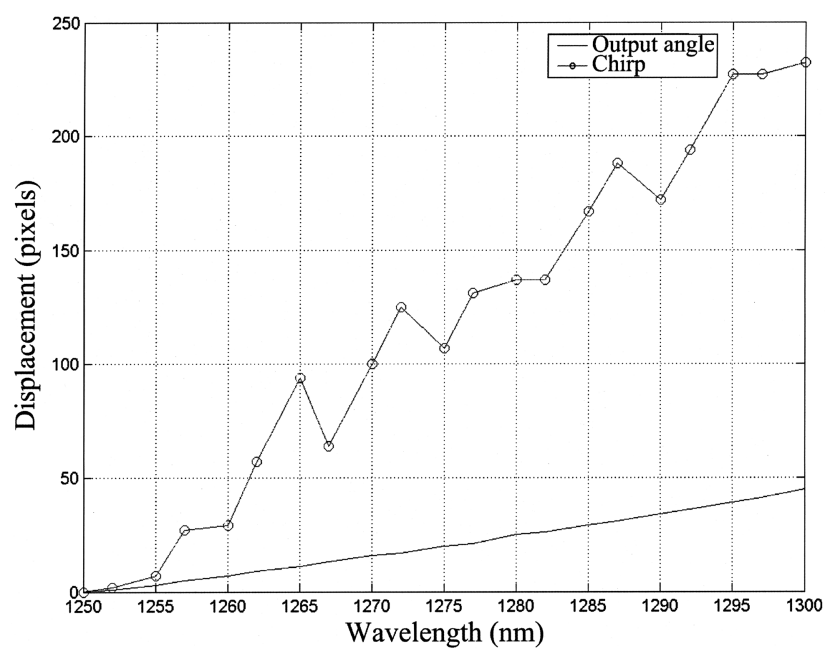

Fig. 3. Comparison between the spatial shift due to the wavelength dependence of the radiation's output angle and that due to the chirp rate. 


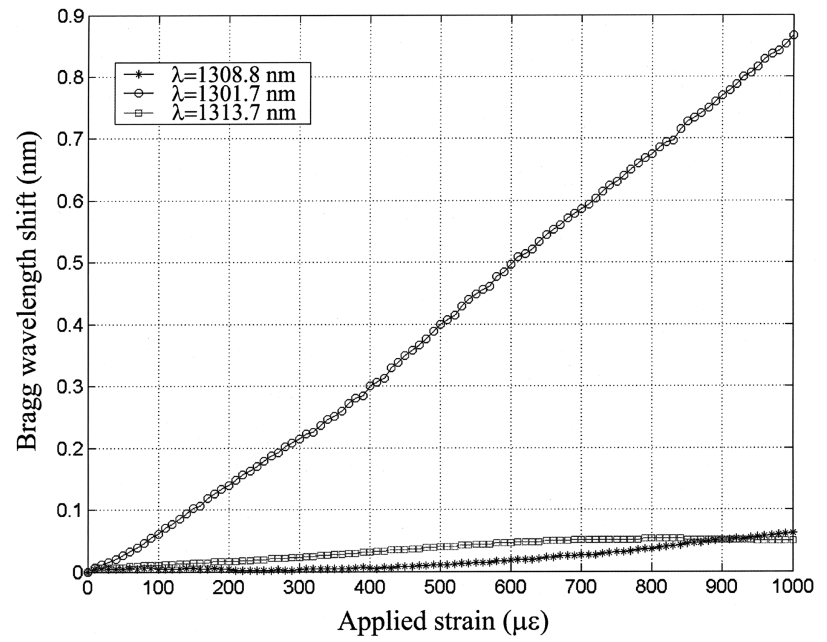

Fig. 4. Response of the proposed interrogation technique to a strain sweep applied to one of the three multiplexed sensing FBGs.
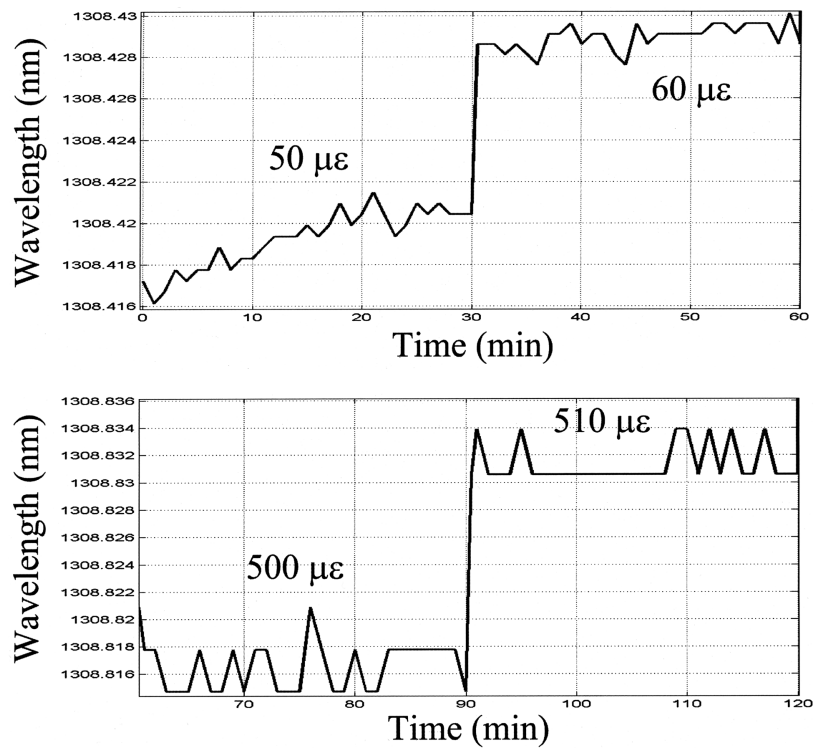

Fig. 5. 1308.5-nm FBG response to the stability experiment.

by a proper configuration of the linear array and by certain modifications of the software.

Another experiment that aimed to test both the stability and the wavelength resolution of the system was carried out, and its results are shown in Fig. 5. The figure shows only the response of the 1308.5-nm Bragg wavelength FBG when the integration time was set to $8 \mathrm{~ms}$ and the analog-digital converter was configured to use 16 bits. The experiment consisted of letting the system evolve during $30 \mathrm{~min}$ at $0-\mu \epsilon$ applied strain and then increasing the elongation in $10 \mu \epsilon$ for another $30 \mathrm{~min}$. At the end of that period the applied strain was raised to $500 \mu \epsilon$, and the cycle was repeated. As can be seen from Fig. 5, both the stability and the noise level are very good. In the first case the drift was $2 \mathrm{pm}$ at most in each of the different 30-min sections (except for the first, which was affected by the warming up of the device). On the other hand, the average noise estimated was $3 \mathrm{pm}$ (this amount can be reduced to less than 2 pm by properly shielding the sensing FBG).

The resolution of the system can be obtained from the same experiment. Accepting that this parameter equals the minimum wavelength shift measured around each $10-\mu \epsilon$ jump, we can establish that the resolution lies within the 0.4 - or the $3.7-\mathrm{pm}$ range depending on the Bragg wavelength. Both values are within the usefulness range (that can be set in $5 \mathrm{pm}$ ), but the first is comparable with the best reported so far of which we are aware.

In summary, in the present Letter a novel interrogation scheme has been reported. It is based on measuring the characteristics of the near-field radiation generated by a chirped tilted fiber Bragg grating when it is illuminated by light coming from sensing FBGs. With such an arrangement a compact interrogation technique suitable for in-field use is obtained. In addition, its characteristic wide wavelength interrogation range, simultaneous interrogation of multiplexed sensing FBGs, good stability and linearity, and wavelength resolution of better than $3.7 \mathrm{pm}$ make this scheme promising in the context of FBG interrogation.

All the work reported here, both theoretical and experimental, including the chirped tilted fiber Bragg grating fabrication, was carried out in the Photonic Engineering Laboratory of the University of Cantabria. This work was cosupported by the Spanish Comisión Interministerial de Ciencia y Tecnología TIC'2001-0877-C02-01 and the Fondos Europeos de Desarollo Regional SISFOCDETIC 1FD97-0775 projects. The proposed scheme is patent pending. C. Jáuregui's e-mail address is jauregui@teisa.unican.es.

\section{References}

1. J. M. López-Higuera, ed., Handbook of Optical Fiber Sensing Technology (Wiley, New York, 2002).

2. A. D. Kersey, T. A. Berkoff, and W. W. Morey, Electron. Lett. 28, 236 (1992).

3. M. A. Davies and A. D. Kersey, Electron. Lett. 30, 75 (1994).

4. A. D. Kersey, T. A. Berkoff, and W. W. Morey, Opt. Lett. 18, 1370 (1993).

5. C. Jáuregui and J. M. López-Higuera, Microwave Opt. Technol. Lett. 37, 124 (2003).

6. C. Jáuregui, A. Quintela, J. Echevarría, O. Ma. Conde, and J. M. López-Higuera, in 15th Optical Fiber Sensors Conference Technical Digest (Institute of Electrical and Electronics Engineers, Piscataway, N.J., 2002), pp. $159-162$. 